\title{
Canadian Rheumatologists' Perspectives on Moderate Psoriatic Arthritis and Oligoarticular Psoriatic Arthritis
}

\author{
Dafna D. Gladman ${ }^{1}$ (D), Michael Starr², Alfred Cividino ${ }^{3}$ (D), Anne-Julie Gaudreau ${ }^{4}$, Jennifer Jelley ${ }^{4}$, \\ Denise Nicholson ${ }^{4}$, and Jacob Karsh ${ }^{5}$ (i)
}

\begin{abstract}
Objective. Psoriatic arthritis (PsA) substantially impairs quality of life. Clinical trials generally focus on polyarticular PsA, but less is known about the assessment and management of oligoarticular and moderate PsA. An online survey was conducted to determine Canadian rheumatologists' perspectives on the definition and treatment of oligoarticular and moderate PsA.

Methods. Regional and national experts treating patients with PsA were asked to complete an online survey to assess their approach to identifying and managing patients with PsA. Survey questions were developed based on guidance from a committee of Canadian rheumatologists.

Results. Sixty-four of 78 rheumatologists responded, representing 6 major Canadian provinces. Nearly half of respondents were in practice $>20$ years. The majority of rheumatologists reported using swollen joint count (SJC) to describe moderate PsA (86.4\%) and oligoarticular PsA (96.7\%), and considered location of inflammation in PsA assessments. SJC cutoff scores for reporting moderate PsA varied among rheumatologists, suggesting lack of an agreed-upon definition for moderate PsA. Sixty-eight percent of rheumatologists identified access to treatment as the greatest challenge with oligoarticular PsA.

Conclusion. According to the surveyed rheumatologists, SJC remains a key assessment variable when defining oligoarticular and moderate PsA. Although the number of joints is considered when determining the effect of PsA on patients, joint location and functional impairment are also considered when describing the disease as moderate. Access to treatment for patients with $<5$ affected joints is challenging.
\end{abstract}

Key Indexing Terms: disease severity, oligoarthritis, psoriatic arthritis, rheumatologists, survey, treatment

Psoriatic arthritis (PsA) is a chronic inflammatory musculoskeletal disease that leads to impaired health-related quality of life and physical function. ${ }^{1,2}$

This study was funded by Celgene. Amgen acquired the worldwide rights to Otezla (apremilast) on November 21, 2019. Celgene had a role in the study design, the collection, analysis, and interpretation of the data, and the revision of the manuscript. Amgen Inc. had a role in revising the report and in the decision to submit the report for publication.

${ }^{I}$ D.D. Gladman, MD, Schroeder Arthritis Institute, Krembil Research Institute, Toronto Western Hospital, Toronto, Ontario ${ }^{2}$ M. Starr, MD, McGill University Health Center, Montreal, Québec; ${ }^{3}$ A. Cividino, MD, McMaster University Faculty of Health Sciences, Hamilton, Ontario; ${ }^{4}$ A.J. Gaudreau, $M S c$, J. Jelley, BScN, D. Nicholson, BSW, Amgen Inc., Mississauga, Ontario; 5 J. Karsh, MDCM, FRCPC, The Ottawa Hospital, Ottawa, Ontario, Canada.

$D D G$ has received grant/research support and served as a consultant for AbbVie, Amgen Inc., Bristol Myers Squibb, Celgene, Janssen, Novartis, Pfizer, and UCB. MS has served as a consultant for AbbVie, Amgen Inc., Celgene, Janssen, Lilly, Novartis, Pfizer, and UCB. AC has received grant/research support from and served as a consultant for AbbVie, Bristol Myers Squibb, Celgene, and Pfizer. AJG, JJ, and DN are employees of Amgen Inc. JK has received honoraria from AbbVie, Celgene, Eli Lilly, and Novartis/Sandoz, and served as an external consultant to the Canadian Agency for Drugs and Technology in Health (CADTH).

Address correspondence to Dr. D.D. Gladman, Toronto Western Hospital, 399 Bathurst St., IE-410B, Toronto, ON M5T 2S8, Canada.

Email:dafna.gladman@utoronto.ca.

Accepted for publication May 5, 2021.
A number of PsA screening tools have been developed to address the challenges associated with identifying patients with PsA, including the Psoriatic Arthritis Screening Evaluation (PASE), Toronto Psoriatic Arthritis Screen (ToPAS), and Psoriasis Epidemiology Screening Tool (PEST). ${ }^{3}$

Variables commonly used to assess PsA in the clinic are the assessment of tender and swollen joints either using the 68/66 joint count or a reduced joint count using the 44 or 28 joint count. According to the Outcome Measures of Rheumatology Clinical Trials (OMERACT) and the Group for Research and Assessment of Psoriasis and Psoriatic Arthritis (GRAPPA), the 68/66 joint count should be used in patients with PsA. 45,6 By current definition, polyarticular PsA affects $\geq 5$ joints and oligoarticular PsA affects $\leq 4$ joints. ${ }^{5}$ In an observational cohort study conducted in Canada, oligoarthritis was commonly reported in patients with minimal (41\%) and nonminimal (32\%) disease activity. ${ }^{7}$

Although increased joint involvement in PsA is generally associated with greater disease burden, the presence of manifestations such as enthesitis and dactylitis can also increase the effect of the disease on patients. ${ }^{1,8}$ In an analysis of data from the national database of the German Collaborative Arthritis Centres, patients with oligoarticular PsA reported higher disease burden compared with patients with polyarticular PsA, despite having lower disease activity and severity. ${ }^{9}$ The study found a similar prevalence of axial symptoms, enthesitis, dactylitis, and 
comorbidities between the 2 PsA subtypes, ${ }^{9}$ suggesting oligoarticular PsA may be as complex as polyarticular PsA.

Articular symptoms of PsA are commonly managed with nonsteroidal antiinflammatory drugs, corticosteroid injections, and conventional/nonbiologic and biologic disease-modifying antirheumatic drugs (DMARDs), with biologic options recommended for patients with polyarticular or severe PsA. ${ }^{10,11}$ Consequently, an unmet need exists for an effective treatment for patients with oligoarticular PsA. ${ }^{8}$ In fact, evidence from the Multinational Assessment of Psoriasis and Psoriatic Arthritis Survey indicates that PsA is undertreated overall, with $59 \%$ of patients with suspected PsA receiving no treatment or topical treatment only. ${ }^{12}$

The majority of PsA data are sourced from US and European studies; thus, the prevalence, treatment, and challenges of Canadian patients with PsA are not well understood. We aimed to evaluate the Canadian rheumatologists' definitions for the terms oligoarticular Ps $A$ and moderate Ps $A$, and to gain perspective on the categorization of disease severity, unmet needs, and treatment relative to both definitions.

\section{METHODS}

Rheumatologist survey. The Canadian PsA Perspectives Survey was conducted from April 24, 2018, through December 9, 2018. Regional and national Canadian rheumatology experts in PsA management were invited to complete an online survey to assess their approach to identifying and managing patients with PsA. These rheumatologists with known expertise in the management of PsA are considered regional and national opinion leaders. The survey consisted of 22 questions and was developed based on feedback from a steering committee of Canadian rheumatologists. Survey topics included demographic information for participating rheumatologists, identification of PsA, assessments of disease severity, challenges facing patients with PsA, and proportion of patients receiving treatment from one of the PsA therapy classes. The survey questions are available in the Supplementary Data (available with online version of this article).

Survey participants completed the survey using Survey Monkey software (Survey Monkey) before participating in an industry-sponsored focus group. Survey results were summarized descriptively and reported as mean, median, and range ( $\min , \max )$ for continuous outcomes and percentage of rheumatologists for categorical outcomes. To help elucidate the differences in perception and definition of the terms moderate Ps $A$ and oligoarticular $P_{S} A$ when used to describe a patient subset, participants completed 1 of 2 versions of the survey, with 1 version using the term moderate Ps $A$ and the other version using the term oligoarticular Ps $A$.

This online survey was noninterventional and did not involve patients, nor did it have any investigative sites. As such, ethics board approval was not applicable and was not required. In addition, rheumatologists voluntarily participated in the survey.

\section{RESULTS}

Respondents. A total of 64 rheumatologists representing 6 major Canadian provinces responded to the survey; the actual number of responses was $<64$ for some questions if a respondent skipped a question (Supplementary Table 1, available with the online version of this article). Overall, almost half of the respondents were community-based, 23.6\% were hospital-based, and 29.1\% were mixed. Twenty-six (47.3\%) of the respondents reported being in practice for $>20$ years. The majority of rheumatologists surveyed were not involved in clinical trials; of those who were clinical trialists, more than one-third were involved in 1-3 concurrent clinical trials. The version of the survey that used the term moderate Ps $A$ was completed by 31 respondents before September 16, 2018; after this date, 33 respondents completed the second version of the survey that used the term oligoarticular PsA. Overall, no substantial differences in responses were observed between respondents who completed the survey using the term moderate PSA and those who completed the survey using the term oligoarticular PsA.

Variables used to assess PsA. When evaluating patients with moderate or oligoarticular PsA, the majority of rheumatologists reported they would use swollen joint count (SJC) as an assessment parameter (Figure 1). When asked which aspects of moderate PsA were important to rheumatologists and patients, respondents ranked the presence of enthesitis and dactylitis as having the same importance as SJC. Rheumatologists further indicated that they also consider the location of inflammation in their assessment of PsA, particularly inflammation in the knees, ankles, wrists, fingers, toes, and lower back. When asked to consider all aspects of oligoarticular PsA that were important to rheumatologists and patients, respondents identified SJC, followed by enthesitis and dactylitis, as key manifestations they would use when describing a patient with oligoarticular PsA. Other indicators used in the definition included tender joint count (TJC), work productivity, patient perception, and Health Assessment Questionnaire (HAQ) score.

Defining Ps A variables. Rheumatologists were asked to define the ranges of SJC, TJC, enthesitis, and dactylitis that best classify patients with moderate or oligoarticular PsA. The overall SJC range was broad, with a lower and upper cutoff mean (median) of 3.5 (3.0) and 10.1 (6.0), respectively, for moderate PsA (Figure 2) and 2.7 (2.0) and 9.3 (5.0), respectively, for oligoarticular PsA (Figure 3). The mean (median) cutoff for defining severe PsA with SJC was $5.8(6.0)$ in the survey that used the term moderate $P_{s} A$ and $8.8(6.0)$ in the survey that used the term oligoarticular $P_{s} A$, according to respondents. Similarly, a broad range of cutoff scores were reported for TJC, enthesitis, and dactylitis (Figure 2, Figure 3).

Rheumatologist-identified patient challenges. When asked about the greatest challenges for Canadian patients with moderate PsA, rheumatologists chose access to treatment as the most challenging, followed by patient acceptance, presence of comorbidities, treatment choices, diagnosis, when to initiate treatment, and lack of treatments (Supplementary Figure 1, available with the online version of this article).

Ps $A$ treatment. For the treatment of patients with moderate and oligoarticular PsA, the surveyed rheumatologists reported using mainly conventional DMARDs. Biologic medications were identified as being more frequently used for severe PsA than for moderate PsA or oligoarticular PsA (Supplementary Table 2, available with the online version of this article).

\section{DISCUSSION}

Despite the high prevalence and chronic nature of PsA, ${ }^{11,13,14}$ the needs of patients with oligoarticular PsA are currently 


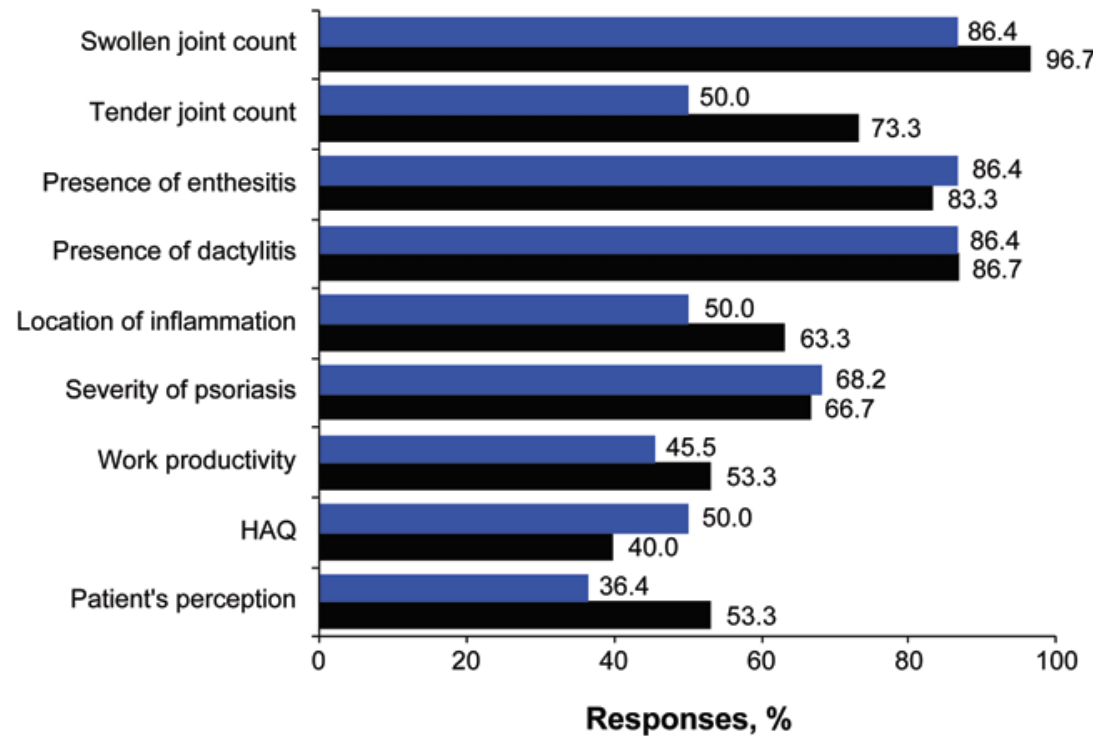

\section{Survey version:}

Moderate PsA

(22 respondents)

- Oligoarticular PsA

(30 respondents)

Locations of inflammation that best define moderate and oligoarticulat PsA

\begin{tabular}{|lcc|}
\hline & Moderate & Oligoarticular \\
\hline Fingers & $85.0 \%$ & $65.5 \%$ \\
Wrists & $90.0 \%$ & $69.0 \%$ \\
Toes & $55.0 \%$ & $62.1 \%$ \\
Knees & $85.0 \%$ & $95.6 \%$ \\
Ankles & $80.0 \%$ & $86.2 \%$ \\
Lower back & $45.0 \%$ & $6.9 \%$ \\
\hline
\end{tabular}

Figure 1. Variables used to assess PsA. Rheumatologists $(\mathrm{n}=52)$ responded to the question: When considering all aspects of the disease that are important for you and your patient, what would you use to describe a patient who has moderate/oligoarticular psoriatic arthritis? HAQ: Health Assessment Questionnaire; PsA: psoriatic arthritis.

A

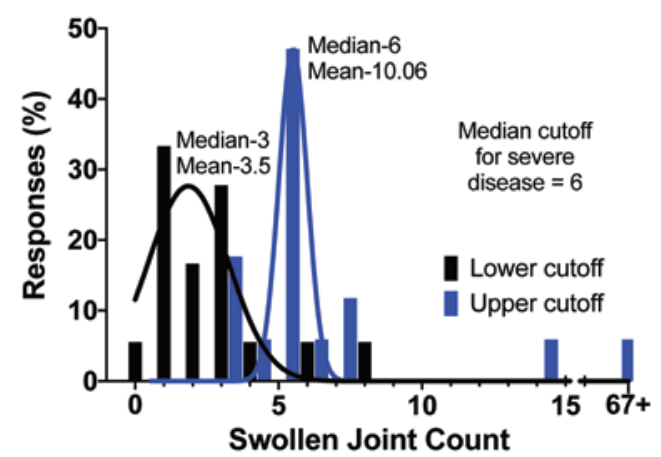

C

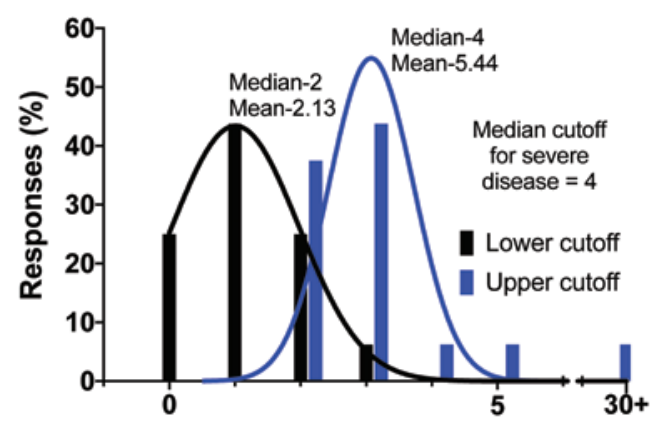

Enthesitis
B

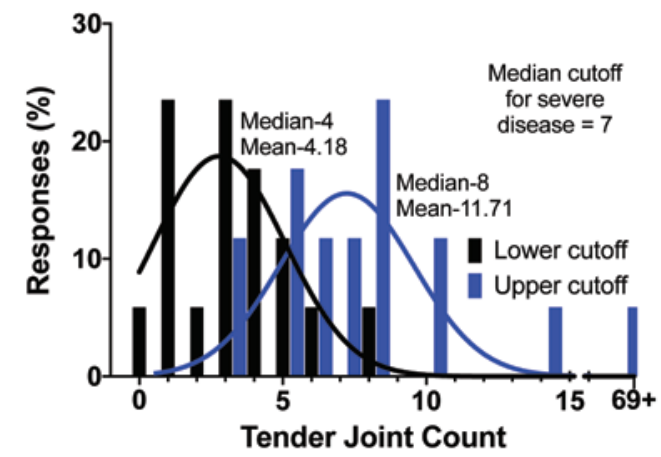

D

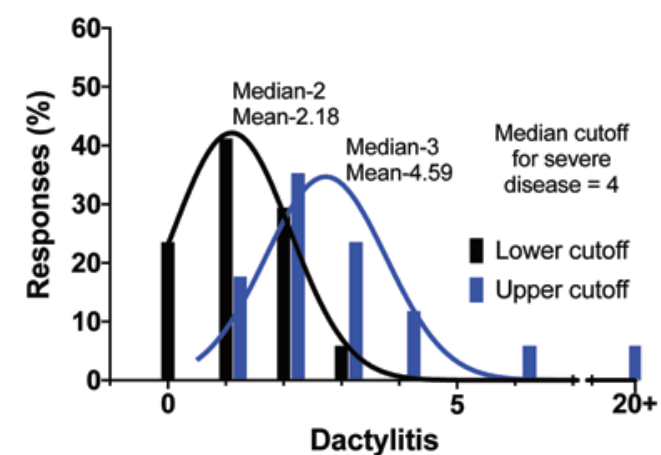

Figure 2. Rheumatologist-reported assessment scale cutoffs for patients with moderate PsA. Low and high cutoff scores reported by rheumatologists ( $\mathrm{n}=18$ for all) for (A) swollen joint count; (B) tender joint count; (C) enthesitis; and (D) dactylitis. The $\mathrm{n}$ values reflect the number of rheumatologists responding to the question: Please define the range that best classifies a patient with moderate PsA. PsA: psoriatic arthritis.

not widely recognized. ${ }^{8}$ Indeed, defining disease domains and assessing disease severity are the most important steps toward addressing the needs of patients. We applaud the most recent
American College of Rheumatology and European League Against Rheumatism (European Alliance of Associations for Rheumatology) guidelines for suggesting treatment algorithms 
A

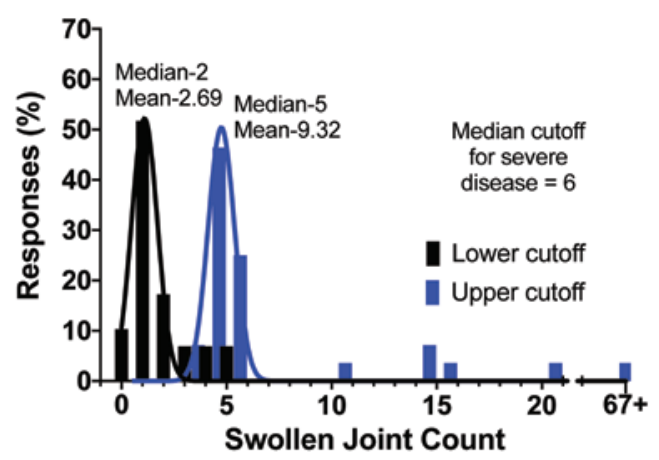

C

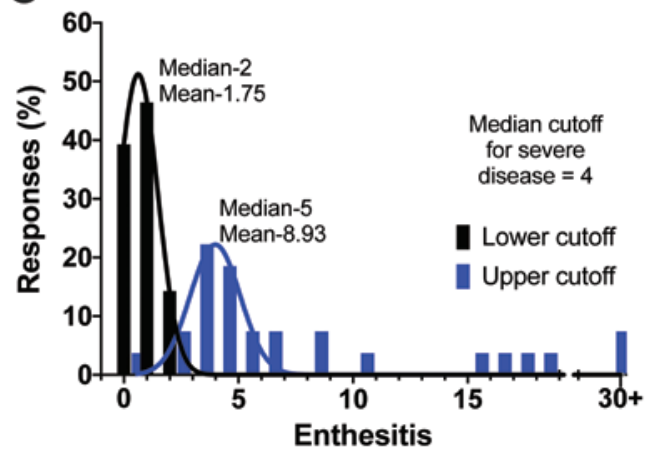

B

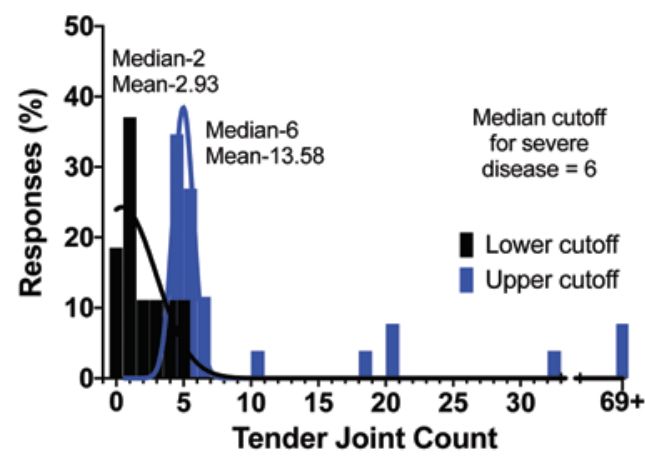

D

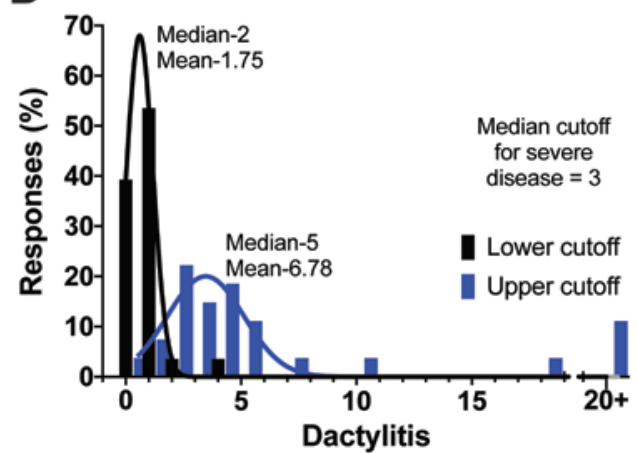

Figure 3. Rheumatologist-reported assessment scale cutoffs for patients with oligoarticular PsA. Low and high cutoff scores were reported by rheumatologists ( $\mathrm{n}=29$ for all) for (A) swollen joint count; (B) tender joint count; (C) enthesitis; and (D) dactylitis. The $n$ values reflect the number of rheumatologists responding to the question: Please define the range that best classifies a patient with oligoarticular PsA. PsA: psoriatic arthritis.

that consider patient preferences as well as disease severity presenting as moderate vs severe PsA, ${ }^{11}$ or oligoarticular vs polyarticular joint involvement. ${ }^{15}$

A large majority of surveyed rheumatologists reported SJC as the measure they use when defining moderate PsA, along with TJC, enthesitis, dactylitis, work productivity, patient perception, and HAQ score. When specifying the lower and upper SJC cutoffs for defining moderate PsA, the surveyed rheumatologists reported a wide range of scores, suggesting a lack of agreement on the definition of the broader term moderate PsA. Agreement on the definition of moderate PsA may be lacking because patients with few actively inflamed joints could be considered to have severe disease depending on the location of the joints involved and the impact of disease on the individual patient. On the other hand, rheumatologists' responses were more consistent when using the term oligoarticular $P_{s} A$, likely because this term has a well-established clinical definition. ${ }^{8} 16$ However, more efforts are needed to ensure definitions of disease severity are used consistently in rheumatology and dermatology routine clinical care.

The surveyed rheumatologists also reported their perspective on the greatest challenges facing Canadian patients with oligoarticular PsA. Approximately two-thirds of rheumatologists reported access to treatment as a greater challenge than patient acceptance, presence of comorbidities, treatment choices, diagnosis, when to initiate treatment, and lack of treatment.
This finding is consistent with prior surveys of physicians and patients in which financial and insurance issues presented challenges when considering treatment options for patients with PsA. ${ }^{17,18}$ Because current clinical trials, which may not include many patients with oligoarticular PsA, largely focus on polyarticular PsA, and treatment guidelines acknowledge the lack of strong data regarding the treatment of oligoarticular PsA, little is agreed upon about the management of oligoarticular PsA., ${ }^{8,10,11,15}$ The lack of clear guidance on the treatment of oligoarticular PsA contributes to difficulties in obtaining insurance authorizations, which can limit access to appropriate treatments for these patients. From the Canadian perspective, provincial formularies will not reimburse the cost of biologics for patients with $<5$ swollen joints, denying the highest tier of treatment for patients with oligoarticular PsA. ${ }^{19}$

The surveyed rheumatologists reported low overall systemic medication use among Canadian patients with PsA. Prior surveys of physicians and patients with PsA showed significant concerns regarding the tolerability and safety of DMARDs. ${ }^{12,18}$ Considering that methotrexate may not be an effective treatment option for managing patients with enthesitis and dactylitis, ${ }^{20}$ no ideal options are currently available to address some of the specific needs of patients with moderate PsA or oligoarticular PsA. GRAPPA recommends tailoring PsA treatment based on domain involvement ${ }^{10}$ highlighting the need for rheumatologists 
to consider other PsA domains when choosing treatment, such as enthesitis, comorbidities, and patient preference, in order to optimize patient benefit.

Our study has several limitations. The survey included Canadian rheumatologists who agreed to participate in a Celgene-sponsored steering committee or advisory board and may not be fully representative of the broader community of Canadian rheumatologists. As with other surveys, the current study may be subject to recall bias and differences in how rheumatologists interpreted the questions. The design of this online survey did not allow for responses to be clarified. We did not consider using the Disease Activity Index for Psoriatic Arthritis (DAPSA) $^{21}$ or Psoriatic Arthritis Disease Activity Score $\left(\right.$ PASDAS ${ }^{22}$ to define moderate disease. The DAPSA relies predominantly on joint counts to define disease activity and is more suited for polyarticular disease. Although the PASDAS has been shown to work well for oligoarthritis, ${ }^{23}$ it does not consider the presence of spondylitis and is not often used in clinical practice.

We found that defining moderate PsA and oligoarticular PsA elicits somewhat different responses. Moderate PsA is not only defined by SJC but also encompasses other manifestations of PsA, with their relative severity and impact on patients. Oligoarticular PsA is defined primarily by joint count, with reduced emphasis on severity of other manifestations. This discrepancy suggests a need to decouple treatment access from joint count and instead focus on the impact on individual patients, which includes assessing for all features of PsA, as well as patient-reported outcome measures.

Oligoarticular PsA and moderate PsA present a challenging scenario with many outstanding questions: What are the best ways to identify and measure active disease? What are the appropriate treatment goals and treatment options with proven efficacy? To close these gaps, more research is needed, with a focus on the assessment tools for routine clinical use, goals incorporating the patient's perspective, and population data examining phenotype-specific outcomes.

\section{ACKNOWLEDGMENT}

Writing support was funded by Celgene and Amgen Inc. and provided by Kristin Carlin, RPh, MBA, of Peloton Advantage, LLC, an OPEN Health company.

\section{ONLINE SUPPLEMENT}

Supplementary material accompanies the online version of this article.

\section{REFERENCES}

1. Kavanaugh A, Helliwell P, Ritchlin CT. Psoriatic arthritis and burden of disease: patient perspectives from the Population-Based Multinational Assessment of Psoriasis and Psoriatic Arthritis (MAPP) survey. Rheumatol Ther 2016;3:91-102.

2. Husted JA, Gladman DD, Farewell VT, Cook RJ. Health-related quality of life of patients with psoriatic arthritis: a comparison with patients with rheumatoid arthritis. Arthritis Rheum 2001;45:151-8.

3. Coates LC, Aslam T, Al Balushi F, Burden AD, Burden-Teh E, Caperon AR, et al. Comparison of three screening tools to detect psoriatic arthritis in patients with psoriasis (CONTEST study). Br J Dermatol 2013;168:802-7.
4. Coates LC, Mumtaz A, Helliwell PS, Mease PJ, Callis-Duffin K, Krueger GG, et al. Development of a disease severity and responder index for psoriatic arthritis (PsA) -- report of the OMERACT 10 PsA Special Interest Group. J Rheumatol 2011;38:1496-501.

5. Coates LC, FitzGerald O, Gladman DD, McHugh N, Mease P, Strand V, et al. Reduced joint counts misclassify patients with oligoarticular psoriatic arthritis and miss significant numbers of patients with active disease. Arthritis Rheum 2013;65:1504-9.

6. Duarte-Garcia A, Leung YY, Coates LC, Beaton D, Christensen R, Craig ET, et al. Endorsement of the $66 / 68$ joint count for the measurement of musculoskeletal disease activity: OMERACT 2018 psoriatic arthritis workshop report. J Rheumatol 2019;46:996-1005.

7. Coates LC, Cook R, Lee KA, Chandran V, Gladman DD. Frequency, predictors, and prognosis of sustained minimal disease activity in an observational psoriatic arthritis cohort. Arthritis Care Res 2010;62:970-6.

8. Marchesoni A. Oligoarticular psoriatic arthritis: addressing clinical challenges in an intriguing phenotype. Rheumatology Ther 2018;5:311-6.

9. Huscher D, Albrecht K, Bischoff S, Thiele K, Behrens F, Fischer $\mathrm{K}$, et al. Patients with psoriatic arthritis and oligoarthritic subtype report higher disease burden than patients with a polyarthritic pattern - data from the German collaborative arthritis centres [abstract]. Arthritis Rheumatol 2015;67:679.

10. Coates LC, Kavanaugh A, Mease PJ, Soriano ER, Laura Acosta FM, Armstrong AW, et al. Group for Research and Assessment of Psoriasis and Psoriatic Arthritis: treatment recommendations for psoriatic arthritis 2015. Arthritis Rheumatol 2016;68:1060-71.

11. Singh JA, Guyatt G, Ogdie A, Gladman DD, Deal C, Deodhar A, et al. Special article: 2018 American College of Rheumatology/ National Psoriasis Foundation Guideline for the Treatment of Psoriatic Arthritis. Arthritis Care Res 2019;71:2-29.

12. Lebwohl MG, Bachelez H, Barker J, Girolomoni G, Kavanaugh A, Langley RG, et al. Patient perspectives in the management of psoriasis: results from the population-based Multinational Assessment of Psoriasis and Psoriatic Arthritis Survey. J Am Acad Dermatol 2014;70:871-81.

13. Gladman DD, Antoni C, Mease P, Clegg DO, Nash P. Psoriatic arthritis: epidemiology, clinical features, course, and outcome. Ann Rheum Dis 2005;64 Suppl 2:ii14-7.

14. Zachariae H. Prevalence of joint disease in patients with psoriasis: implications for therapy. Am J Clin Dermatol 2003;4:441-7.

15. Gossec L, Baraliakos X, Kerschbaumer A, de Wit M, McInnes I, Dougados M, et al. EULAR recommendations for the management of psoriatic arthritis with pharmacological therapies: 2019 update. Ann Rheum Dis 2020;79:700-12.

16. Ritchlin CT, Colbert RA, Gladman DD. Psoriatic arthritis. N Engl J Med 2017;376:957-70.

17. Armstrong AW, Robertson AD, Wu J, Schupp C, Lebwohl MG. Undertreatment, treatment trends, and treatment dissatisfaction among patients with psoriasis and psoriatic arthritis in the United States: findings from the National Psoriasis Foundation surveys, 2003-2011. JAMA Dermatol 2013;149:1180-5.

18. van de Kerkhof PCM, Reich K, Kavanaugh A, Bachelez H, Barker J, Girolomoni G, et al. Physician perspectives in the management of psoriasis and psoriatic arthritis: results from the population-based Multinational Assessment of Psoriasis and Psoriatic Arthritis survey. J Eur Acad Dermatol Venereol 2015;29:2002-10.

19. Ontario Ministry of Health. Exceptional access program reimbursement criteria for frequently requested drugs. February 1, 2021. [Internet. Accessed June 1, 2021.] Available from: www. health.gov.on.ca/en/pro/programs/drugs/docs/frequently_ requested_drugs.pdf 
20. Nash P, Clegg DO. Psoriatic arthritis therapy: NSAIDs and traditional DMARDs. Ann Rheum Dis 2005;64 Suppl 2:ii74-7.

21. Schoels MM, Aletaha D, Alasti F, Smolen JS. Disease activity in psoriatic arthritis (PsA): defining remission and treatment success using the DAPSA score. Ann Rheum Dis 2016;75:811-8.

22. Helliwell PS, FitzGerald O, Fransen J. Composite disease activity and responder indices for psoriatic arthritis: a report from the
GRAPPA 2013 meeting on development of cutoffs for both disease activity states and response. J Rheumatol 2014;41:1212-7.

23. Coates LC, Mahmood F, Emery P, Conaghan PG, Helliwell P. The dynamics of response as measured by multiple composite outcome tools in the TIght COntrol of inflammation in early Psoriatic Arthritis (TICOPA) trial. Ann Rheum Dis 2017;76:1688-92. 